\title{
واقع التعليم الجامعي في ضوء اقتصاد المعرفة ومبررات التحول نحو الجامعة المنتجة
}

\author{
إعداد \\ / إسراء محمد احمد محمدل رجب \\ باحثّة دكتور اة \\ كلية التربية بقنا - جامعة جنوب الو ادى
}




\title{
واقع التعليم الجامعي في ضوي اقتمساد المعرفة ومبررات التحول

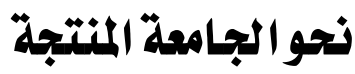

\author{
إعلداد \\ 1/ إسراء محمد احمد محمل رجب \\ باحثة دكتور راة \\ كلية التربية بقنا - جامعة جنوب الو ادى لو
}

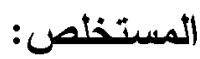

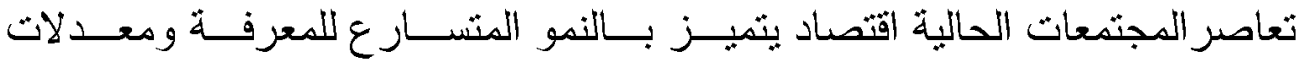
الاكتثافات العلمية و الابتكارات التكنولوجية.حيث تحولت المجنمعات من مجتمعـات صناعية الي مجتمعات قائمة علي المعلومات و المعرفة، فلم بعد رأس المال المـادي هو العامل الاساسي للقوي الاقتصادية، بل اصبح رأس المال المعرفي هــو المحسـد

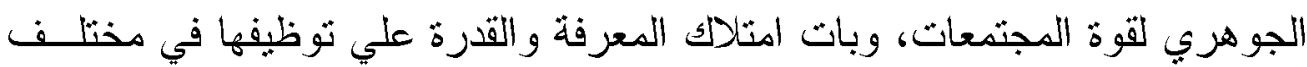
المجالات هو المعيار الرئيسي لقياس نقدم الدول. ويمثل العنصر البشري هو الــرأس المال الفكري الحقيقي لهذا لاقتصاد،لانه هو الذي ينتج ويدير ويوظف المعرفة. غيران

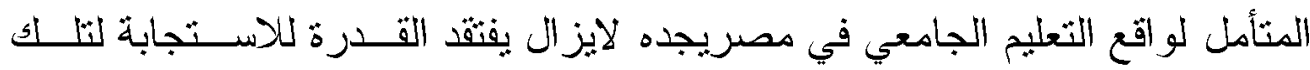
المتغير ات المحلية و العالمية،بالاضـافة الي العديد من التحديات التعليمية و التمويليــة و التكنولوجية الثي يو اجهها وغيرها من المبررات الاقتصادية والاجثماعية الثي دعت

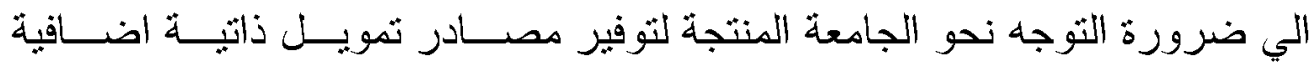
ومعالجة التحديات ومواكبة المتغيرات التي فرضها عصر اقتصاد المعرفة ـ وتنتاول الباحثة فيما يلي و اقع التعليم الجامعي في ضوء اقتصاد المعرفة ومبررات التوجـهـ

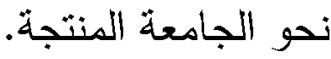

الكثمات المفتاحية: اقتصاد المعرفة؛الجامعة المنتجة؛ التعليم الجامعي • 


\title{
Reality of University Education in the light of Knowledge Economy, and Justifications of shifting towards Productive University
}

\author{
Esraa Mohamed Ahmed Mohamed Ragab \\ PhD Scholar at Department of Education Principles \\ Qena Faculty of Education, South Valley University
}

\begin{abstract}
:
Today's societies are experiencing an economy characterized by the rapid growth of knowledge, the rates of scientific discoveries, and technological innovations.As societies transformed from industrial societies into societies based on information and knowledge, material capital is no longer the main factor for economic power, but knowledge capital has become the essential determinant of the strength of societies. And possessing knowledge with the ability to employ it, in various fields, has become the main criterion for measuring the progress of countries. Thus the human element is the real intellectual capital of this economy, because it is the one that produces knowledge, then manages and employs it.However, the reality of university education in Egypt still lacks the ability to respond to these local and global variables, in addition to the educational, financing and technological challenges that it faces, and other economic and social justifications. All of that called for the need to go towards the productive university to provide additional self-financing sources, address the challenges and keep pace with the changes imposed by knowledge economy.
\end{abstract}

Keywords: economy characterized, productive university, Education university 
مقدمة:

قد شهد القرن الحادي والعشرين تطورا هائلا في كافة المجالات، وأفرزت التكنولوجية الحديثة الكثبر من المتغيرات، واصبحت المعرفة واستخداماتها سمة اساسية من سمات العصر الحالي. ويعتبر التعليم الجامعي المنتج الرئيسي للمعرفة ولرأس المال الفكري، بما ثقوم به من تتقيف وتدريب للطلاب واكسابهم المهارات (Bejinaru,2017,252) اللذزمة

ويلاحظ بثأمل واقع التعليم الجامعي المصري ان مؤسساته نعاني عدة مشكلاث ثقلل من قدرتها علي المنافسة في مجتمع اقتصاد المعرفة. كما ذكرت نتائج دراسة(الثرف

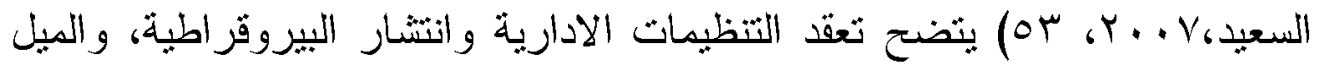
الي التتمبط وجمود المناهج وكل ذلك يفقد الطاقات الفكرية بالتعليم الجاهعي القدرة علي الابتكار والأبداع وفرص التنافس والتميز. كما يعاني نظام التعليم الجامعي في مصر من نقص التمويل وعدم الكفاءة في معظم المستويات والمجالات، كما بعاني من ضعف المخصصات المالية للبحث العلمي وتثني مؤشر جودة مراكز البحث العلمي بالجامعات (الهيئة العامة

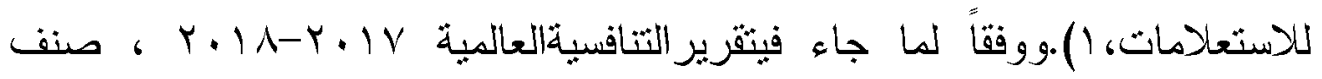
المنتدى الاقتصادي العالمي جودة نظام التعليم في مصر في المرثبة . با بين VIس دولة) Ramage,2019)

لذا أصبح التعليم الجامعي في حاجة إلي فلسفة وطرق تعليمية مثماثية مع العصر المعرفي القائم علي التتافسية في الإنتاج،لتطوير العنصر البشري وتوفير موارد مالية من خلال الإنتاج المعرفي. ومن هنا يمكن التسؤال الأتي ما واقع التعليم الجامعي في ضوء اقتصاد المعرفة ؟ وما هي مبررات التحول نحو الجامعة المنتجة؟ وانطلاقا من 
ذلك ثهدف المقالة الحالية الي دراسة واقع التعليم الجامعي والتحديات التي تواجهه في عصر اقتصاد المعرفة، من اجل فهم ثلك التحديات والمشكلات ومن ثم معالجتها.

\section{أولًا: واقع التعنيم الجامعي في ضوع اقتصاد المعرفة}

يعد اقتصاد المعرفة مصدر للنمو الاقتصادي وتحقيق القدرة التتافسية في جميع القطاعات الأقتصادية.ويعرف اقتصاد المعرفة بأنه الاقتصاد الذي تتنج فيه المعرفة معظم القيمة المضافة، مما بعني ان المعرفة - في هذا الاقتصاد- تعد المكون الاساسي لعمليتي الانتاج والتسويق، ويزداد النمو مع زيادة المعرفة القائمة علي الثقنيات الحديثة لتكنولوجيا المعلومات والاتصالات باعنبارهم المرنكز الرئيسي لهذا

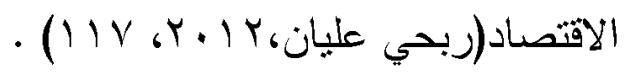

بري الكثير من الباحثني والعلماء ان اقتصاد المعرفة برنكز علي عدة عناصر أساسية تثمثل في؛وسائل الاتصال و التكنولوجيا الحديثة، الابتكار، الثنبكات، الثوزيع الجيد، الثقنيات و العمالة المهرة (-951 -Harris,2011,913) .ويشه التعليم الجامعي المصري -حاليا- بكافة انماطه وعملياته ونظمه العديد من التحديات التي تحد من قدرثه علي الثواكب مع عصر اقتصاد المعرفة ، وتعجزه عن الثواجد في صدارة سباق المنافسة الاقليمية والعالمية، ويمكن استقراء واقع التعليم الجامعي المصري و استتباط اهم تحدياته ومشكلاته علي النحو الاتي: ا- تعديات المدخلات وضعف المخرجات للتعبيم الهامعي: بدراسة و اقع التعليم الجامعي المصري من خلال تحليل مدخلاته ومخرجاته بتضح انه يعاني دن تدني مدخلاته ومخرجاته وقصور في العملية التعليمبة واداءها، وقد

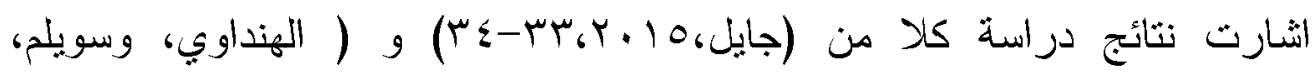
ء (.ب، 10) الي عدم تتاسب مخرجات الثعليم مع سوق العمل، وكذلك جمود 
وتخلف المقررات الدراسية وعدم مواكبتها للنطور المعرفي والعلمي.مما بجعل التعليم الجامعي عاجز عن نطوير مؤسسات الانتاج في المجتمع ، كما بعجز عن امداده بالموارد البشرية القادرة علي التكيف في بيئات عمل اقتصاد المعرفة. ويمكن استقراع واقع مدخلات ومخرجات التعليم الجامعي المصري علي النحو الاتهي: 1- 1 :تعدبات مدخلات التعليم الجامعي:

بالنظر تحديداً الي ددخلات العملية التعليمية في التعليم الجامعي؛ يتضح ان هناك ارتفاع هائل في نسب الطلاب الي المدرسين، ويمثل ذلك تحدي لا يسنهان به في

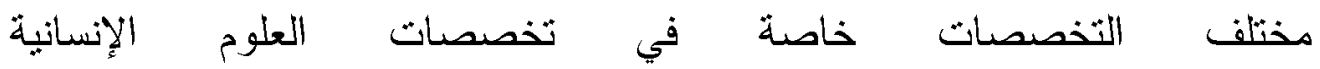
و الاجتماعية(Handoussa,2010,64). و تتشمل التحديات الداخلية للتعليم الجامعي ؛ تحديات متعلقة بالطلاب والاستاذ الجامعي والمناهج والمباني والادارة التعليمبة ويمكن ابضاحها كما يلي: -تحدبات متعلقة بالطلاب:

تثعدد التحديات والمشكلات المتعلقة بالطالب الجامعي ، وثئول بعض الدراسات هذه التحديات الي عوامل ذاتية ونفسية للطالب ، تثمثل في الشعور بالاختلال الوظيفي ، وفقدان الثتة ، واضطر ابات نفسية كالاحباط والقلق والثوثر ويرتفع معدل انتشار الاضطر ابات والمشكلات النفسية لدي الطلاب في البلدان والبيئات والأسر ذات الدخل المنخفض والقفيرة(Kabir,2018,7).

وهناك المشكلات الاكاديمية خاصة في السنة الأولي من الدراسة ونتمثل في؛ صعوبة اسنتيعاب الطلاب للمناهج و المواد الدراسية المكثةة، وضعف الثمكن من المهارات الاساسية للتعلم، واهمال الالتزام لحضور المحاضرات التعليمية(جودة،

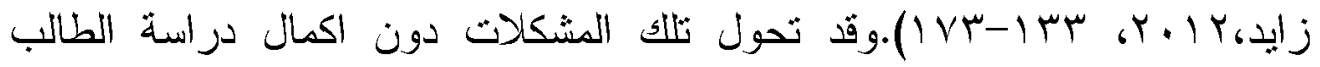


الجامعية او تؤدي الي تعثره اكاديميا كتأخر نخرجه مما ينسبب في ثدني مستوي تحصيله التعليمي والمعدل التز اكمي الدراسي له. -تحديات متعلقة بالمناهج الاربية: تثمثل مشكلات المناهج والمقررات الدراسبة في جمود المناهج الدراسبة والثقادم المعرفي فيها، وتجاهل النكامل المعرفي بين التخصصات. بالاضافة الي اقتصار التعلم علي الكتب الدراسية ونقص النشجيع علي البحث والطلاع والوصول الي مصادر نعلمية اخري، وكذلك جمود نظم الثقويم والامنحانات.وينقق ذلك مع ما اسفرت عنه نتائج دراسة استقصائية (Kraf,2012,6) من افنقار مناهج التعليم الجامعي المصري للمهارات الاساسية والفشل في نزويد الطلاب بخبرات ذات صلة للواقع المهني وسوق العمل، وتجاهل مناهجها لمعالجة قضايا المجتمع. - تعدبات متعلقة بالأستاذ الجامعي:

يعد صعوبة الملائمة بين مهام التدريس والبحث ، احد اهم التحدياث الرئيسية التي يواجها معلمي التعليم الجامعي. حيث تشير نتائج دراسة ميدانية (Neshkovska,2016,37-38) الاساتذة الجامعيين وتمنعهم من نشر المزيد من الاوراق البحثية سنوياً هو العجز المادي واستهلاك نشاطهم التدريسي لمعظم وقتهم وذلك بحسب نسبة r.V\%\% من معلمين الثعليم الجامعي، بينما صرح حوالي · (\% فقط بأنهم لا يجدوا صعوبة في التوفيق بين اجر اء البحوث ومهام الثدريس. وكذلك يعاني معلمي التعليم الجامعي من تحديات مالبة صعبة، قد اشارت نتائج الدراسات الي ان غالبية معلمين التعليم الجامعي بنسبة (10/\%) يعانوا من النقص الخطير في الموارد المالبة الذي يؤثر بالسلب علي حجم ومقدار مثاركتهم ومساهمتهم العلمية في المؤتمرات و النجمعات العلمية(Neshkovska,2016,39). 


\section{-تحديات مخرجات التعليم الجامعي:}

تتجلي تحديات مخرجات التعليم الجامعي في ثدني مستوي مخرجات التعليم الجامعي وعدم ملائمتها لسوق العمل والقطاعات الانتاجية اليوم ، لاتفي العمالة المصرية بجميع منطلبات السوق وهي عائق حاسم أمام نمو القطاع الانتاجي وقدرثة التتافسية ؛ لوجود نقص في التخصصات الحديثة، و اقتثار التدريب العملي(Handoussa,2010,41).ينتج عنه ضعف المهارات الانتاجية المطلوبة لقطاعات العمل والانتاج ، وبالتالي لا يجد المهارات المدربة التي يمكن الاعتماد عليها مما يدفع نحو الاستعانة بعمالة اجنيية مدربة وذات مهار ات. r - تحدي التمويل مع تزايد الطلب علي التعلبيم الجامعي: بدراسة واقع التعليم الجامعي المصري يثضح ان التعليم الجامعي في مصر يواجه تحديا خطيرا يتعلق بأزمة تمويله ونقص الموارد المالية له مقارنة بحجم الطلب عليه.ومن صور تحديات التمويل التي تواجه التعليم الجامعي في مصر؛ التمويل غير الكافي للإنتاج المعرفي؛ حيث اثشارت نتائج دراسة (Loveluck,2012,14) الي ارتفاع حدة تحدياث الثمويل في مصر نظرا لما مرث به مصر من فترة انتقالية صعبة، استغرقت من الدولة فترة طويلة للعودة الي المسار الصحيح وجمع الأموال مرة أخري.

\section{r-تنـي القدرة التنافسية وفقًا للتصنبفات العالمبية:}

يتضح بالرجوع الي ثقارير التصنيفات العالمية للجامعات حول العالم؛ وجود تدني واضح للجامعات المصرية في القرة علي المناقسة ، يمكن عرض وثثييم واقع

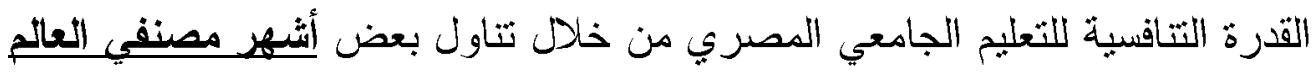
للجامعات وذلت علي النحو التالي: 
- تصنيف جامعة جياو ثونج شانغهاي ARWU طبقاً لقائمة شنغهابي لأفضل . . جامعة عالمبا ، قد ثمكنت جامعة مصرية حكومية واحدة فقط ان نتافس للاخول في قائمة افضل .0 جامعة ، ولكن جاء نرثنبها مندني جدا. ويثضح ذلك من الجدول : الثالي (ARWU, 2020)

\begin{tabular}{|c|c|c|}
\hline & \multicolumn{2}{|c|}{ ARWU ranking تصنيف جياو ثونج شانغهاي } \\
\hline Year السنة & University الجامعة & Rank التصنيف \\
\hline$Y .10$ & جامعة القاهرة & $\varepsilon .1-0$. \\
\hline$r .19$ & جامعة القاهرة & $\varepsilon .1-0$. \\
\hline Y.IV & جامعة القاهرة & $\varepsilon .1-0$. \\
\hline$r \cdot \mid \wedge$ & جامعة القاهرة & $\Sigma .1-0$. \\
\hline$r .19$ & جامعة القاهرة & r. $1-0 .$. \\
\hline
\end{tabular}

وكما هو موضح في الجدول أعلاه؛ ثبرز جامعة القاهرة كجامعة حكومية واحدة تتافست بقوة مع جميع الجامعاث حول العالم للحصول علي مكان في تصنيف

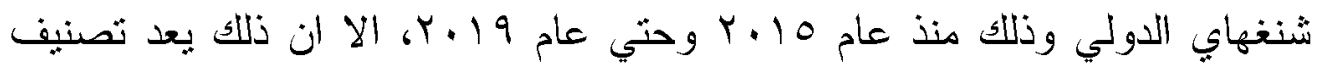
مندني جدا فلم تصل بعد جامعة مصرية واحدة الي تصنيف من فئة افضل مئة جامعة في تصنيف شنغهاب • - تصنيف كيو أس للجامعات العالمية THES_QS World University

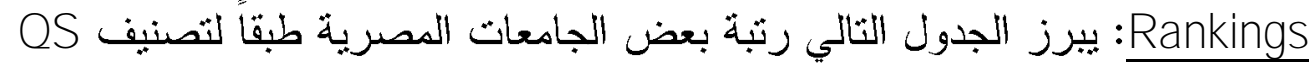
لأفضل . .0 جامعة حول العالم(QS World University Rankings): 
واقع التعليم الجامعي في ضوء اقتصاد المعرفة ومبررات التحول نحو الجامعة المنتجة

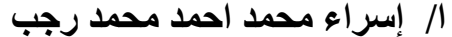

\begin{tabular}{|c|c|c|}
\hline & \multicolumn{2}{|c|}{ QS ranking تصنيف كيو أس } \\
\hline Year السنة & University الجادعة & Rank التصنيف \\
\hline$Y .10$ & الجامعة الامريكية بالقاهرة & rq. \\
\hline$r \cdot 17$ & الجامعة الامريكية بالقاهرة & $r \leq 0$ \\
\hline$r \cdot I V$ & الجامعة الامريكية بالقاهرة & mo \\
\hline$r \cdot 1 \wedge$ & الجامعة الامريكية بالقاهرة & $\begin{array}{c}\text { wo } \\
\leqslant q \cdot-\leq \lambda 1\end{array}$ \\
\hline$r+19$ & الجامعة الامريكية بالقاهرة & $\varepsilon r$ \\
\hline
\end{tabular}

ويتضح من الجدول السابق ان الجامعة الامريكية بالقاهرة وهي ثعد جامعة خاصة كانت نثنافس بقوة للحصول علي نزثبب اكاديمي وفقاً لتصنيف QS لأفضل .. جامعة علي مدار الخمس اعوام السابقة بجانب جامعة حكومية واحدة وهي جامعة القاهرة، ولم يثم العثور علي ابي جامعة مصرية من بين افضل ، . ا جامعة.

\section{؛ قَصور الني الادارية والتنظيمية وضعف التخطيط:}

يعاني التحليم الجامعي المصري الكثير من التحديات والقصور في هياكله النتظيمية والادارية وكذلك نخبط في رؤي التخطيط الاستر اتيجي السليم الأمر الذي يضعف مرونة التعليم الجامعي ويبطء من قدرنه علي سرعة الاستجابة لمتطلبات

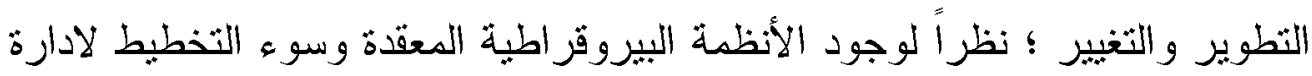
المعرفة بصورة مو اكبة للتحديات و المتغير ات المحلية و العالمية. ويأتي ذلك وفقا لما اكدته نتائج دراسة ( الحسيني ،ع ا ـ ب، 0 1) الي وجود مشكلات وتحديات ثتمثل في جمود التشريعات المنظمة للتعليم الجامعي، وافتقار الكفايات الادارية الكفء والمؤهلة للقيادة والادارة و التخطيط. وينرثب علي ذلك غلبة النمطية و عدم مواءمة مخرجات التعليم مع منطلبات سوق العمل ، وغياب القدرة التتافسبة نظرا لافثتاد الرؤي المستقبلية والخطط الاستراتيجية اللازمة لتحقيق المزايا و القدرة التتافسية. 
0- جمود التطوير للتواكب مي اقتصاد المعرفة وتدني الاستجابة للمتفيرات المحلية والعالميـة:

فرض الثوجه العالمي نحو اقتصاد المعرفة مهام ومنطلبات حديثة ومنطورة علي الدول والمجنمعاث كي تسنطيع التواجد في بيئة اقتصادية تنافسية منغيرة وسريعة التطور، وتعتبر مؤسسات التعليم الجامعي الأدوات الاساسية للمجتمعاث للتواجد في اقتصاد المعرفة والمنافسة فيه وذلك لما ثقوم به تلك المؤسسات من أدوار هامة في بناء رأس المال المعرفي وتحقيق التمية المستدامة. حيث ثؤكد كثير من الثواهد ان التعليم الجامعي في مصر بعاني من عجز مواءمة منطلبات سوق العمل مع مخرجات التعليم نظر الخلل التخطيط في الموارد التعليمية البثرية وغير البثرية وغياب النطوير وفقا لنقافة الانتاج والاكتفاء بثدريس المقررات الدراسية بعيدا عن ربط العطية التعليمية مع بيئة العمل والانتاج في المجتمع (World Economic Forum report,2011,26-36). ثانياً: مبررات تطوير التعليم الجامعي في ضوع اقتصاد المعرفة والجامعة المنتجة بشهدالعالمالمعاصر عدثنالتحدياتالمتعددة و المتجددة في مختلف المجالات ؛ وبات واضحاً إدراك " الفجوة المعرفية بين الوطن العربي وبين دول العالم المثقدم:لهذا

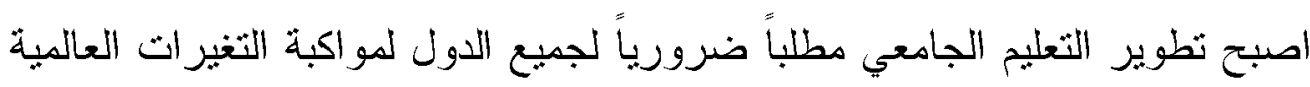
المتسارعة في ظل اقتصاد ثتسار ع فيه التغيرات التكنولوجية و المعرفية. وذلك من خلال ثبني صبيغ جامعية مبنكرة كالتوجه نحو الجامعة المنتجة، ويقصد بالجامعة المنتجة المؤسسة المنتجة قادرة على ثرويج منتوجها العلمي وتقديم خدمات الخبرة والاستشارة و البحث حسب الطلب بما بضمن لها موارد مالية ذاتية قابلة

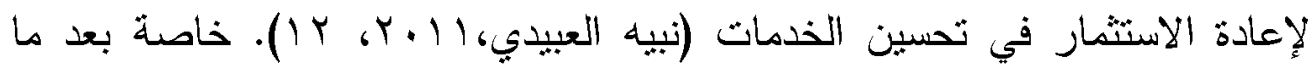
فرضه اقتصاد المعرفة من تحديات متعددة كتحديات الجودة وتحديات انتاج المعرفة و انماط الاتتاج وتحديات السوق العالمي. ويمكن تتاول هذه المبررات فيما يلي: 


\section{1-مبرزرات مهنية وتعليمية:}

يو اجه التعليم الجامعي في مصر واقع ثعليمي لا يعكس منطلبات العصر و لا ثتاسب مخرجاته مع منطلبات سوق العمل وقطاعات الاعمال الانتاجية واقتصاد المعرفة.قد رصدت الهيئة القومية لضمان جودة التعليم والاعنماد بعض النقاط السليية المهنية التي تحد من كفاءة اداء التعلبم الجامعى وتعد مبررا قويا للتحسين و النطوير ومنها ما

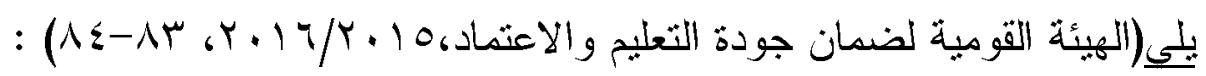
- عدم اهثمام بعض المؤسسات بمراجعة لوائح مراحل البكالوريوس والدراسات العلبا في ضوء المعايير الأكاديمية القومية، لتحديد حجم الفجوة واجراء التعديلات المطلوبة.

- عدم اهنمام اغلب البر امج بإدراج مقومات الثوظف ضمن نواتج التعلم المستهذفة. والقصور في إكساب الطلاب المهارات المهنية المستهفة من خلا المقررات

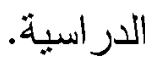
- عدم اتباع المؤسسات للتوجهات الحديثة في التعليم العالي عامة والجامعي - علي وجه الخصوص - في مجال تتمية البرامج التعليمبة لمهارات ريادة الأعمال. - محدودية دور وحدة ضمان الجودة في تطوير ومثابعة وتتفبذ خطط دعم القدرة المؤسسبة. - انخفاض نسبة الطلاب الوافدين الملتحقين بالدراسة في مرحلتي البكالوريوس

$$
\text { و الدر اسات العليا. }
$$

- ضعف الميز انية المخصصة للبحث العلمي كوالعجز في إيجاد مصادر ثمويل بديلة للبحث العلمي حيث بعتمد أغلبها علي الموازنة المخصصة من الدولة. - ضعف إنتاجية البحث العلمي،وقلة عدد بر اءات الاختر اع المنتجة علي مستوي كل كلية وعدم الاستفادة من نتائج البحث العلمي في نطوير المقررات الدراسية. 
- ضعف نسويق البحث العلمي وضعف الثراكات مع الصناعة في مجال البحث العلمي، وقصور تتمبة الموارد الذاتية في مؤسسات التعليم الحكومية.

\section{ب}

ثواجه مجثمعات العالم المعاصر ظروف اقتصـادية سريعة التغير وشديدة الثتافس في ظل اقتصاد يقوم علي علاقاث مثرابطة بين قطاعات الانتاج و الصناعة ومؤسسات التعليم الجامعي، مما دفع المؤسسات الجامعية الي السعي نحو التطوير، من خلال ثبني نموذج الجامعة المنتجة للتكيف مع المبررات الاقتصادية المتعددة المصاحبة لعصر اقتصاد المعرفة، ويمكن تتاول تلاك المبررات علي النحو التالي:

r-1 :محدودية التمويل:تثمنل ازمة الثمويل في التعليم الجامعي المصري في كون الثمويل الحكومي هو المصدر الرئيسي لتمويل مختلف الأنثطة الخاصة بالتعليم الجامعي في مصر • وتسهم الدولة في تمويل التعليم الجامعي الحكومي من خلال الجامعات بنسبة نتراوح من ه>\% الي ، \% ، في حين بترك للجامعات ومؤسسات التعليم العالي الحكومية مهمة ثوفير الجزء المنبقي (· (10uropean (1\%) بشكل ذاتي من خلال استراتيجيات منعددة ومختلفة .(Commission, 2010,5 r-Y البطالة:وتعني هدر لر أسمال بشري غير مستغل. لا تكمن خطورة البطالة من الارتفاع الحالي لمعدلاتها وتفاقم نتائجها الراهنة ، بل في ثوقع زيادنها في المستفبل. وثبلغ نسبة العاطلين عن العمل من فئة الجامعيين وفوق الجامعيين الي ا.ـابـ طبقا

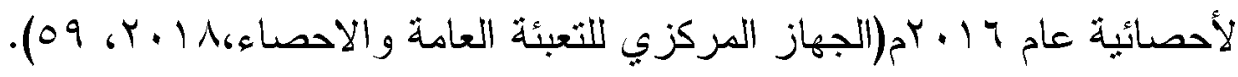
r-r :غياب الرؤية الاقتصادية وتدنى معدل الانفاق العام على التعليم الجامعي: تجسدت ابرز ملامح غياب الررؤي الاقتصادية في مصر والنتائج المترنبة عليها، 


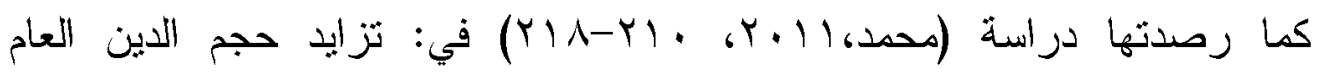
الخارجي، وارتفاع مؤشر التضخم، ونراجع معدلات الاستشار وتدني معدلات النمو الاقتصادي.مما تسبب في ضعف قدرة الدولة علي تلبية المطالب وتغطبة الاحتياجات الاساسية لمؤسساتها وتدني معدلاث الانفاق علي الثعليم.

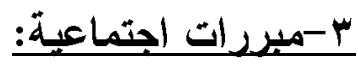

قد طرء علي المجتمعات العديد من التحديات الاجنماعية الثي اثرت علي نسيجه وتكوينه الاجنماعي وكذلك مثلت تحدي لمؤسساته التعليمبة خاصة التعليم الجامعي ومن هذه التحديات ما بلي: r-1 :عدم التكافؤ الاجنماعي و الققر

تؤثر الظروف الاقتصادية والازمات المالية الني يمر بها المجتمع علي نسبة التعليم الجامعي لبعض الفئات الاجتماعية دن النساء والطبقة الفقيرة والمتوسطة فالتحو لات الاقتصادية والتعليمية تجعل التعليم الجامعي يمثل عبئًا مالياً علي الأسر

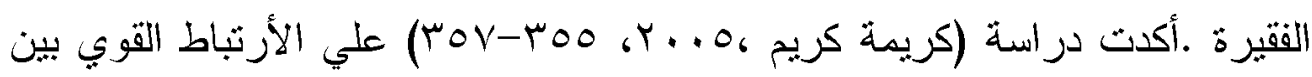
حالة الفقر ومستوي التعليم ، وأن التحليم هو أول مايتم الاستغناء عنه عند هبوط الاخل للأسر الفقيرة ،ومن ثم تكون فرص الابناء اقل في الحصول علي التعليم.

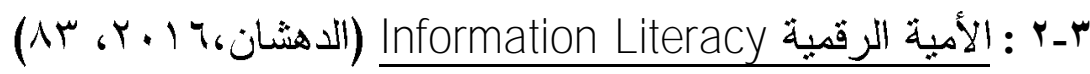
تعد الأمية الرقمية أخطر التحديات التي ثواجه المجتمات ، وتعرقل اي محاو لات في سبيل التنمية والنطوير، ففهوم الأمبة في هذا العصر لم يعد يقتصر علي عدم قدرة الثخص علي القراءة والكتابة، بل تخطي ذلك الي البعد الرقمي، واصبحت الأمية في عصر اقتصاد المعرفة تتمثل في عدم قدرة الفرد علي الاستخدام والتعامل مع معطيات العصر الرقمي وثقنياته. 


\section{Brain Drain هجرة العقول أو الكفاءعات}

تعثبر هجزة العقول او الكفاءات من اعضاء هيئة التشريس والخبراء مثكلة تعرقل

التعليم الجامعي في كل من الدول النامية والمثقدمة علي السواء، ومن ضمنهم

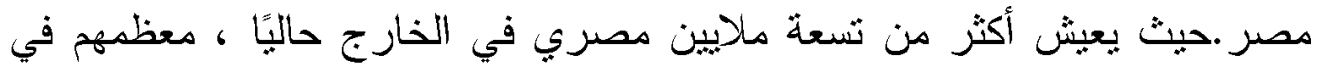
دول الشرق الأوسط وشمال إفريقيا الأخرى، ولكن أيضًا في الولايات المتحدة وكندا

$$
\begin{aligned}
& \text { وأوروبا وأسنر اليالون (Ramage,2019). }
\end{aligned}
$$

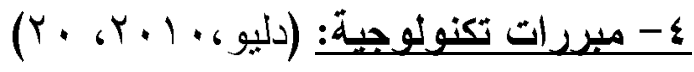

ينمثل الثحدي التكنولوجي امام التعليم الجامعي في القدرة علي الاستفادة من التكنولوجيا في تطويعها في تحسين مستوي التعليم الجامعي وادارة وتتظيم النتاج الفكري. وذلك لان استخدام النكنولوجيا اصبح من الاساسيات المهمة في عصر اقتصاد المعرفة لثطوير التعليم الجامعي نحو الإنتاج. ويتحقق من خلال رفع قدرة المستفبدين من التكنولوجيا الحديثة سواء اعضاء هيئة تدريس او طلبة أو ادارة،من خلال التدريب المستمر و المتابعة الدائمة وتبادل الخبرات والار اء و المعرفة في مجال

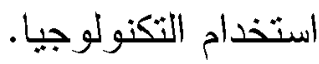

\section{الخغلاصة:}

تتاولت المقالة بإيجاز تحليلا مجملا لأهم وأبرز التحديات التي تواجه التعليم الجامعي في مصر في ضوء اقتصاد المعرفة ونيان مفهومه ودعائمه، ومن ثم عرضت بعض لمض المبررات و الدو اعي الجوهرية الثي ثنتضي ضرورة الثوجه نحو الجامعة المنتجة لمواكبة عصر اقتصاد المعرفة، ويتشنتج هما سبق ما يلي: - أن اقتصاد المعرفة بعتمد في المقام الاول علي استخدام الأفكار، وعلي نطبيق التكنولوجيا.ويعتبر استخدام الموارد الحديثة مثل تكنولوجيا التعليم، ثقنيات المعرفة و إنتاج المعلومات، و العمال ذوبي المهارات العالية من العوامل الاساسية المكونة و المحركة لأقتصاد المعرفة. 
بالرغم من ان ظهور اقتصاد المعرفة أكد علي أهمبة التعليم الجامعي كمرنكز رئيسي لإنتاج المعرفة وإعداد عمال المعرفة،لاتز ال الجامعات المصرية منخفضة في انتاجية المعرفة. حيث تم العثورعلي عدد قليل من الجامعات المصرية في التصنيف الدولي ولكن بثرثيب متذني جدا، ولم يثم العثورعلى جامعة مصرية من بين أفضل . . . أبي تصنيف عالمي.

- فرضت كثير من الدواعي والمبررات ومنها المهنية منل؛ تدني مخرجات التعليم الجامعي وتدني الجودة وجمود المناهج، ودواعي اقتصادية مثل؛ ازمة الثمويل والبطالة وتدني معدل الانفاق، وبعض الدواعي التكنولوجية المنمثلة في الثقنيات التكنولوجية المتجددة وصعوبة نوظيفها وثورة المعلومات، فرض كل ذلك حتمبة التوجه نحو نبني نموذج الجامعة المنتجة. 


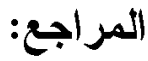

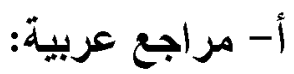

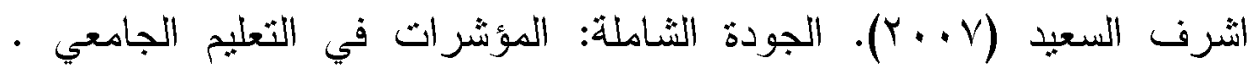
الأسكندرية :دار الجامعةالجديدة.

الهيئة القومية لضمان جودة التعليم والاعتماد(10 • ب/ 1 • ب).الكتاب السنوي للههيئة القومية لضمان جودة التعليم والاعتماد.مصر : رئاسة مجلس الوزراء. الهنداوي، باسر فتحي و سويلم، محمد غنبم(ك ( ب). استر اتيجية مقترحة لتجسير الفجوة بين مخرجات التعليم الجامعي واحتياجات سوق العمل بمصر في ضوء

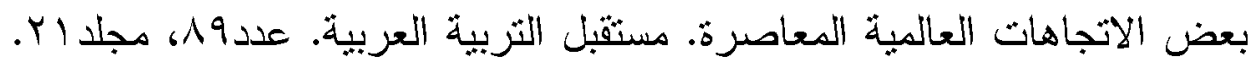
المركز العربي للتعليم و التتمية. ابريل. مصر •

الحسيني،عزةاحمد(ع ( ب). التفكير الاسنرانيجي لدي قادة التعليم الجامعي المصري علي ضوء بعض النماذج و النطبيقات الاجنبية. مجلةالنزبية. عددلى. مجلد ا ا فبر اير ـ الجمعية المصرية للتربية المقارنة والإدارة التعليمبة.

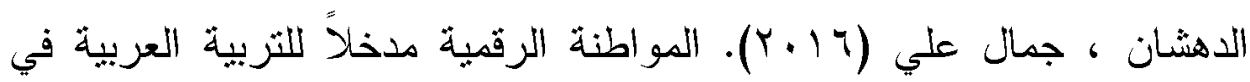
العصر الرقمي. مجلة نقد وتتوير •ع ه.الفصل الثاني- السنة الثانية ـ قرطبة . اسبانبا.

جودة ،يسري محمد وز ايد ،احمد احمد (Y (Y. (Y). المثكلات الاكاديمية ونوعيثها من وجهة نظر طلاب كلية التربية بجامعة حائل. مجلة العلوم التربوية. مجلد( (Y). القاهرة. الجهاز المركزي للتعبئة العامة والاحصاء (1) (Y). مصر في ارقام. مارس. جمهورية مصر العربية. 
جايل ، عفاف محمد(10 ب). التخطيط الاستراتيجي لتتمبة مهارات خريجي التعليم الجامعي لمواجهة المنطلبات المتجددة لسوق العمل فيضوء اقتصاد المعرفة، مستثبل التربية العربية. عدد9ه،مجلد بr. . يوليو • مصر . فضيل دليو(·(r). التكنولوجيا الجديدة للإعلام والاتصال، المفهومالاستعمالات- الاقاق. الأردن: دار الثقافة.

عليان، ربحي مصطفي(Y (Y) (Y). اقتصاد المعرفة. عمان: دار صفاء اللنشر والتوزيع. كريمة كريّم(0 . . ب). دراسات في الفقر و العولمة: مصرو الدول العربية، ثرجمة سمير كريم. القاهرة: المجلس الاعلي للثقافة. محمد ، سماح زكريا(1) (1). منطلبات تطبيق نظام الجودة والاعتماد في التعليم الجامعي - مع النطبيق علي جامعة بنها. اطروحة دكثوراة. قسم اصول التربية. كلية التربية. جامعة بنها.

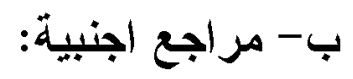

Bejinaru.R.(2017). Universities in the Knowledge Economy.Management Dynamics in the Knowledge Economy. Faculty of Management (SNSPA), Vol.5 no.2; DOI 10.25019 European Commission. (2010). Higher Education in Egypt, National Tempus Office Egypt.

Handoussa.H,et. al. (2010).Situation Analysis: Key Development Challenges Facing Egypt. Cairo Agenda for Action report Sponsored and Published by UNDP to measure the Aid Effectiveness of the Development Partners Group (DPG) to develop a Mutual Strategy for Development Cooperation.

Harris, R.G. (2011). Models of regional growth: Past, present and future. Journal of Economic Surveys. 
AshrafulKabir.A. (2018). A Study on Common Psychological Problems in Intermediate College Students in the Perspective of Banglades, Submitted to Health Research, itspoa Journal,V.1, 15 January.

Kraf.C. (2012). Challenges facing the Egyptian education system: Access, quality, and inequality, Survey of Young People in Egypt Policy Brief no. 2. Cairo: Population Council.

Loveluck.L. (2012). Education in Egypt: Key Challenges, Middle East and North Africa Programme, Chatham House ,March.

Neshkovska.S. (2016). CONTEMPORARY CHALLENGES OF HIGHER EDUCATION TEACHERS , Quality of university learning and teaching Conference proceedings / BrdopriKranju, Ljubljana, 6 April , ISBN 978-961-6628-50-1 .

World Economic Forum report. (2011). in collaboration with Booz \& Company: Accelerating Entrepreneurship in the Arab World, Jordan: Booz \& Company, 21-23 October.

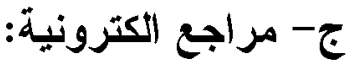

$$
\begin{aligned}
& \text { - الهيئة العامة للاستعلامات: المؤسسات العلمية البحثية. مناح علي: } \\
& \text { www. sis.gov.eg }
\end{aligned}
$$

-نبيه العبيدي (11 ـ ب). اسنر اتيجية التمويل للجامعات المنتجة( جامعات المملكة العربية السعودية ومملكة البحرين - أنموذجاً). مجلة الاكاديمبة العربية في /https://www.ao- journal.org : الدنمارك. عدد • ( نسخة الكترونية ).متاح علي - ARWU World University Rankings 2020. Available on:www.shanghairanking.com

- Ramage Y. (2019). Education in Egypt.Available on :https://wenr. wes.org/

- QS World University Rankings. Available on:/www.qs.com/rankings 